\title{
E Research Square Effects of rooting powder and soil salt stress on the
growth and physiological characteristics of Tamarix chinensis cuttings
}

Jia Sun

Shandong Agricultural University

Jiangbao Xia ( $\nabla$ xiajb@163.com )

Shandong Provincial Key Laboratory of Eco-Environmental Science for Yellow River Delta https://orcid.org/0000-0001-8633-0171

\section{Ximei Zhao}

Binzhou University

\section{Li Su}

Shandong Agricultural University

Chuanrong Li

Shandong Agricultural University

\section{Fanglei Gao}

Binzhou University

Ping Liu

Binzhou University

\section{Research article}

Keywords: soil salt stress, rooting powder, growth, physiological and biochemical indices, Tamarix chinensis

Posted Date: November 18th, 2019

DOI: https://doi.org/10.21203/rs.2.17393/v1

License: (c) (i) This work is licensed under a Creative Commons Attribution 4.0 International License. Read Full License 


\section{Abstract}

Background: Vegetation restoration is a main ecological remediation technology for greening saline and alkaline soils. The objectives of this study were to determine whether aminobenzotriazole (ABT), a rooting powder, can be used to improve the physiological regulatory abilities of Tamarix chinensis under salt stress; to reveal the physiological regulatory pattern by which $\mathrm{T}$. chinensis pre-treated with ABT adapts to salt stress.

Results: (1) As the salt stress level increased, the cutting survival rate, height, and root length of T.

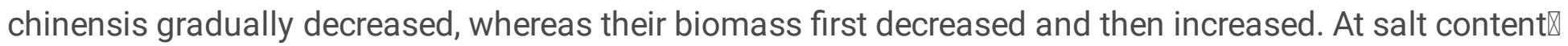
$S \mathrm{c} \>0.9 \%$, cutting propagation of $\mathrm{T}$. chinensis was difficult, and there was a considerable decrease in its biomass. The effectiveness of $A B T$ in improving the survival rate and growth of $\mathrm{T}$. chinensis cuttings became increasingly pronounced as the salt stress level increased. (2) T. chinensis was found to adapt to salt stress through increased $\mathrm{Chl}$ content. However, excess salt stress inhibited $\mathrm{Chl}$ synthesis. ABT can be used to widen the range of tolerance of $\mathrm{T}$. chinensis seedlings to salt stress during Chl synthesis. (3) T. chinensis can eliminate excess reactive oxide species (ROS) by enhancing SOD and POD activities. An excess accumulation of ROS will impede the increase in enzyme activities. ABT can help improve T. chinensis seedling enzyme system regulation and was found to be most effective at a concentration of $100 \mathrm{mg} \cdot \mathrm{L}-1$. (4) ABT can reduce MDA accumulation and damage caused by membrane lipid peroxidation (MLP). ABT at a concentration of $100 \mathrm{mg} \cdot \mathrm{L}-1$ was found to be highly effective in reducing MDA content.

Conclusions: ABT was effective in improving the survival rate and the growth and physiological regulatory abilities of $\mathrm{T}$. chinensis cuttings under salt stress. ABT enhanced the resistance of $\mathrm{T}$. chinensis to salt stress. However, under high S c $(>0.9 \%)$ and ABT concentration (>100 $\mathrm{mg} \cdot \mathrm{L}-1)$ conditions, the physiological regulatory ability of T. chinensis seedlings exposed to salt stress weakened. At S c of $0.9 \%$, T. chinensis seedlings pre-treated with $A B T$ at $100 \mathrm{mg} \cdot \mathrm{L}-1$ exhibited the most vigorous growth, highest biomass, and highest physiological and biochemical regulatory abilities.

\section{Background}

Soil salinization exerts a significant impact on sustainable agricultural development and environmental quality. Crop losses caused by salinization in irrigated areas around the world are estimated at USD 11 billion and continue to rise[1]. Soil salinization is a major environmental risk resulting from natural or human activities. Currently, more than one billion ha of land worldwide is salinized, accounting for $30 \%$ of the total land area[2]. According to the Second National General Survey conducted by the Ministry of Agriculture of China[3], approximately 36 million ha of land in China is salinized, accounting for $4.88 \%$ of China's usable land area. Additionally, 9.209 million ha of farmland is salinized, accounting for $6.62 \%$ of China's total farmland area. In China, salinized soils are distributed across a vast area, primarily in its northwestern, northern, northeastern, and coastal regions. Soil salinization will lead to an increase in the osmotic pressure of the soil solution and decreases in the air and water permeability and the nutrient 
availability in soil. Moreover, soil salinization can also hinder normal plant growth and cause severe vegetation degradation[4]. Conventionally, there are two main approaches for ameliorating saline and alkaline soils. One approach is to directly ameliorate soils. Engineering measures, such as constructing ditches to drain salts, pumping freshwater to reduce soil salinity (Sc), and creating raised fields, are primarily implemented to reduce $S c$ and improve the physical and chemical properties of soils to make them suitable for development of agricultural and forestry industries. However, these measures are costly and have low land-use efficiency. Moreover, it is difficult to implement these measures in areas that lack freshwater resources. The other approach is to employ conventional breeding methods and modern bioengineering measures to breed salt-tolerant crop varieties and to use advanced biotechnologies to improve the salt tolerance of plants. Vegetation restoration measures focusing on planting of local halophytic or salt-tolerant plants have become an important approach for ameliorating saline and alkaline soils[5]. Researchers in China and elsewhere have investigated the use of halophytic plants to improve saline and alkaline soils. Some halophytic plants have been found to be capable of removing salts from soil by absorbing and accumulating the salts[6,7]. Halophytic plants can improve the physical and chemical properties of a soil by reducing its bulk density and improving its porosity. Improvements in the physical and chemical properties of a soil can in turn lead to an increase in its permeability and therefore facilitate salt leaching[8]. However, a main issue in implementing vegetation measures to improve saline and alkaline soils is the difficulty associated with adaptation of the planted seedlings to salt-alkali stress during the initial growth stage, resulting in slow root system growth of the seedlings and a relatively low survival rate. Hence, there is an urgent need to develop methods to improve the survival rate of halophyte seedlings during the initial planting stage and to study their physiological adaptability.

As global soil salinization becomes increasingly prominent, an increasing number of studies are being conducted to examine the physiological and ecological mechanisms by which plants respond to salt stress. These studies focus mainly on crops, vegetables, and specific halophytic plants $[4,9,10]$. The results of these studies are of important guidance value to the breeding of halophytic plants and their application in saline and alkaline soil amelioration and vegetation restoration. Some researchers have found a significant increase in the superoxide dismutase (SOD) and peroxidase (POD) activities and malondialdehyde (MDA) content in the leaves of bean seedlings[11] and Jerusalem artichoke[12] with Sc. Hu et al.[13]reported an increase in the MDA content and SOD and POD activities of Panicum virgatum L. with the salt-alkali stress level. Li et al.[14] found that salt stress inhibited the growth of the branches and roots of Salix matsudana Koidz. Additionally, they also found that low-level salt stress induced an increase in the SOD, POD, and catalase (CAT) activities, whereas high-level salt stress inhibited the activities of antioxidant enzymes. Moreover, they reported that low-level salt stress led to an increase in the MDA content and that the MDA content increased rapidly as the salt stress level increased. Hong et al. found that as the sodium chloride $(\mathrm{NaCl})$ concentration increased, there was a decrease in the relative water content, chlorophyll (Chl)-a (Chla) content, total Chl (ChIT) content, and Chla/Chlb ratio in the leaves of Salix spp., but an increase in their Chlb, proline (Pro), and MDA contents[15]. Additionally, they detected an increase in the SOD activity and soluble protein content in the leaves of Salix spp. under mild salt stress and a significant decrease in these two parameters under moderate and severe salt stress. 
However, Zhu et al. found that as the salt stress level increased, the SOD and POD activities and MDA content in the leaves of $T$. chinensis cuttings first increased and then decreased[16]. Evidently, the physiological and biochemical indices of plants vary relatively significantly with the plant species and salt stress level.

T. chinensis is a shrub or small tree species belonging to the genus Tamarix in the family Tamaricaceae. T. chinensis has a developed root system and a relatively high capacity to break winds, fix sand, preserve water and soil, and ameliorate soil. Owing to its relatively high tolerance to salts, alkalis, and droughts, $T$. chinensis is often used as a primary vegetation restoration tree species for restoring and rebuilding degraded ecosystems in China's western arid and desert regions, Yellow River Basin, and coastal saline and alkaline soil regions[17]. T. chinensis shrubs are the main protective shrubs for muddy coastal zones in saline and alkaline soil regions in the Yellow River Delta. These shrubs play a vital role in maintaining ecosystem stability and improving saline and alkaline soils in coastal zones. In recent years, as a result of global warming, there has been a decrease in precipitation and an increase in evaporation in the Yellow River Delta. Additionally, seawater intrusion caused by natural factors (e.g., ocean current movements) and human activities (overexploitation of underground brine resources) has intensified soil salinization and degraded the $T$. chinensis shrub ecosystem in the Yellow River Delta. Therefore, there is an urgent need to develop saline and alkaline soil amelioration measures that focus on $T$. chinensis shrub restoration. Determining the physiological and biochemical regulation processes by which $T$. chinensis tolerates salts is a key link and essential precondition for T. chinensis shrub restoration. Currently, Chinese researchers study $T$. chinensis in areas such as morphology and taxonomy[18], ecological characteristics[19], cytogenetics[20], and physiological and ecological characteristics under salt[17], saltalkali[21], and salt-drought intercross stress[16]. By contrast, researchers elsewhere, mostly treating $T$. chinensis as an invasive species, investigate its role in ecosystemsas well as biological control and physical removal methods for controlling its growth and propagation[22-24]. The physiological adaptability of $T$. chinensis to salt stress and its role in ameliorating saline and alkaline soils in some regions have also been examined[25].

Aminobenzotriazole (ABT), a rooting powder, is an efficient, broad-spectrum plant growth regulator. By enhancing and regulating the endogenous hormone contents and important enzyme activities of plants, ABT can regulate their metabolism intensity, improve the activity of their root systems, promote their growth, and enhance their tolerance, thereby increasing their survival rate and productivity. ABT has been extensively used in afforestation, seedling transplantation, and cutting propagation. Paradikovic et al. found that a commercial rooting powder played an active role in the rooting and development of Salvia officinalis L. and Rosmarinus officinalis $\mathrm{L}$. cuttings and significantly improved their morphological properties (e.g., plant height, number of leaves, root length, fresh weight, and dry weight)[26]. Song et al. found that Patrinia rupestris cuttings pre-treated with 2,685 and 5,370 $\mu \mathrm{m}$ naphthalene acetic acid or $4,920 \mu \mathrm{m}$ indole-3-butyric acid (IBA) exhibited the best rooting traits (rooting percentage, number of adventitious roots, root length, and fresh weight)[27]. Jamal et al. found the best survival rate and growth conditions in stem cuttings of Clerodendrum splendens pre-treated with $20 \%$ IBA[28]. Some researchers have also examined the use of rooting powders in vegetative propagation. Research has shown that prior 
to cutting propagation, treating current-year low-lignified branches with a rooting powder can significantly improve their survival rate. Tan found that treating T. chinensis shoot cuttings with ABT at $200 \mathrm{mg} \cdot \mathrm{L}^{-1}$ for 1 hour could increase their rooting percentage (to $84.44 \%$ on average) and biomass[29]. A combination of a rooting powder and fertilizer was found to significantly improve root activities in $T$. ramosissima in various soil layers[30]. However, the available studies focused primarily on propagation under single-salt or salt-free conditions, and consequently their results cannot be satisfactorily applied under field conditions. Relatively few studies have been conducted to examine vegetative propagation of $T$. chinensis under various salt stress conditions. Moreover, the effects of rooting powders at various concentrations on the growth and physiological and biochemical characteristics of $T$. chinensis cuttings under salt stress have yet to be investigated. As a result, it is difficult to determine the rooting powder concentrations for $T$. chinensis cuttings under various salt stress conditions in practice.

Considering these findings, in this study, a rooting powder, $A B T$, was used at four concentrations to facilitate the growth of $T$. chinensis cuttings at four simulated Sc levels. T. chinensis cuttings under no salt stress were selected as the control. The $T$. chinensis cuttings were analysed to determine their growth indices (e.g., survival rate, height, root length, and biomass) and their physiological and biochemical indices (e.g., Chl content, SOD and POD activities, and MDA content). The growth conditions and physiological and biochemical characteristics of the $T$. chinensis seedlings pre-treated with $A B T$ at various concentrations under salt stress were investigated. The present results can provide technical support for vegetative propagation techniques for $T$. chinensis and greening of saline and alkaline soils.

\section{Materials And Methods}

\section{Experimental materials and design}

Branch cuttings were harvested from the T. chinensis shrub obtained from the wild in the Shandong Changyi National Marine Ecology Special Reserve; the provenance originates from Changyi City, Shandong Province, China. We comply with the Convention on the Trade in Endangered Species of Wild Fauna and Flora. In mid-February 2018 before T. chinensis began to sprout, T. chinensis branches with a diameter of approximately $1 \mathrm{~cm}$ were cut and harvested. The branches were then sectioned into $15-\mathrm{cm}-$ long cuttings. Each cutting was sectioned obliquely at the base and flat at the top. Four Sc stress levels were studied, namely, mild (0.3\%), moderate (0.6\%), and severe (0.9\% and $1.2 \%)$. An Sc of $\leq 0.1 \%$ was selected as the control (CK). Each soil Sc was prepared based on the dry soil weight. The Sc was monitored once every 7 days, and additional salt was added to maintain the previously established Sc. An 8-cm-deep tray was placed beneath each pot. The water that leaked from each pot into the tray was poured back into the pot. Additionally, the tray was washed, and the washing water was also poured into the pot. This procedure prevented salt loss. The bases of the cuttings were soaked at a depth of $2-3 \mathrm{~cm}$ in ABT solutions of various concentrations $\left(0,50,100\right.$, and $\left.200 \mathrm{mg} \cdot \mathrm{L}^{-1}\right)$. Subsequently, the cuttings were planted in pots filled with soil at each Sc level. Ten cuttings were planted in each pot. Three repeats were performed for each treatment. A total of 600 cuttings were planted in 60 pots. During the initial cutting propagation stage, the pots were watered with freshwater twice a day to maintain a soil water content at 
$60 \%-70 \%$ of the field capacity. After 90 days of propagation, the growth (e.g., biomass), physiological, and biochemical indices of the T. chinensis cuttings were measured and analysed.

\section{Measurement of indices}

Measurement of growth indices: Three pots were selected for each treatment. Five T. chinensis cuttings were selected from each pot to measure their growth indices. The height of each plant was measured using a metre ruler. The aboveground and underground biomass of each $T$. chinensis seedling was measured by harvesting the whole plant. Each whole seedling was dug out and cleaned. Then, the branches, trunk, and root system of each seedling were identified. Each seedling was subsequently fixed at $105^{\circ} \mathrm{C}$ for 30 minutes and then dried in an oven at $80^{\circ} \mathrm{C}$ until a constant weight was achieved.

Measurement of physiological and biochemical indices: Three pots were selected for each treatment. Three $T$. chinensis cuttings were randomly selected from each pot. Normally grown, mature leaves of the T. chinensis cuttings in the same area were collected and tested to determine their physiological and biochemical indices. The photosynthetic pigment content in each leaf was measured per unit fresh weight using the ethanol-acetone soaking procedure[31]. SOD activity was measured by nitroblue tetrazolium photoreduction[32]. POD activity was measured by guaiacol colorimetry[33]. The MDA content was measured by thiobarbituric acid colorimetry[34]. At least three repeated measurements of each index were obtained and subsequently averaged.

\section{Data processing}

The experimental data were calculated in Microsoft Excel 2010. Analysis of variance was performed using SPSS 17.0 statistical software to determine the significance of the difference between the experimental data. Multiple comparisons were performed using the least significant difference method.

\section{Results And Analysis}

\section{Growth characteristics of $T$. chinensis cuttings}

Survival rates of $T$. chinensis cuttings

Salt stress exerted a significant impact on the survival rate of $T$. chinensis cuttings pre-treated with various concentrations of $A B T$ (Figure 1). With increasing salt stress, there was a significant decrease in the survival rate of $T$. chinensis cuttings pre-treated with $A B T$ at each concentration. The survival rate of T. chinensis cuttings with each concentration of ABT was $100 \%$ in the CK group. The survival rate of $T$. chinensis cuttings pre-soaked in water at $S c$ of $0.3 \%$ was significantly lower than that in the CK group $(P<0.05)$. However, there was no significant difference in the survival rate between $T$. chinensis cuttings with each concentration of $A B T$ at $S C$ of $0.3 \%$ and those in the CK group ( $P>0.05)$. At $S C$ of $0.3 \%$, there was also no significant difference in the survival rate among $T$. chinensis cuttings with various 
concentrations of $\mathrm{ABT}(P>0.05)$. These findings demonstrate that the $\mathrm{ABT}$ concentration had no significant impact on the survival of $T$. chinensis in the absence of salt stress and had a relatively minor impact on the survival of $T$. chinensis under mild salt stress. At $S C$ of $0.6 \%$, the survival rates of $T$. chinensis cuttings with ABT at $0,50,100,200 \mathrm{mg} \cdot \mathrm{L}^{-1}$ were $40.00 \%, 20.00 \%, 23.40 \%$, and $36.70 \%$ lower than in the CK group, respectively. Additionally, at $S c$ of $0.6 \%$, the survival rates of $T$. chinensis cuttings

with $\mathrm{ABT}$ at 50 and $100 \mathrm{mg} \cdot \mathrm{L}^{-1}$ were significantly higher than $T$. chinensis cuttings with $\mathrm{ABT}$ at $0 \mathrm{mg} \cdot \mathrm{L}^{-1}$ (i.e., water) $(P<0.05)$. This result suggests that salt stress significantly inhibits and that $A B T$ can significantly increase the survival rate of $T$. chinensis. At $S c$ of $0.9 \%$, the survival rate was highest for $T$. chinensis cuttings with $\mathrm{ABT}$ at $200 \mathrm{mg} \cdot \mathrm{L}^{-1}$ (53.30\%). By comparison, the survival rate of $T$. chinensis cuttings pre-soaked in water was only $20.00 \%$, which was significantly lower than that of $T$. chinensis cuttings with $A B T$ at 50 and $200 \mathrm{mg} \cdot \mathrm{L}^{-1}(P<0.05)$. At $S C$ of $1.2 \%$, the survival rate was $0 \%$ for $T$. chinensis cuttings pre-soaked in water and only $6.7 \%$ for cuttings treated with various concentrations of $A B T$.

Biomass of $T$. chinensis cuttings

As the salt stress level increased, the biomass of $T$. chinensis cuttings with each concentration of $A B T$ first decreased, then increased, and then decreased again (Figure $2 A$ ). At SC of $0.9 \%$, the biomass of individual T. chinensis cuttings with $\mathrm{ABT}$ at $100 \mathrm{mg} \cdot \mathrm{L}^{-1}$ reached a peak value $(16.70 \mathrm{~g})$. The biomass of T. chinensis cuttings with $A B T$ at 0,50 , and $200 \mathrm{mg}^{-L^{-1}}$ was $48.57 \%, 48.59 \%$, and $54.69 \%$ lower than that of $T$. chinensis cuttings with $A B T$ at $100 \mathrm{mg} \cdot \mathrm{L}^{-1}$, respectively. At the same $S \mathrm{c}$, there was a significant difference in biomass among $T$. chinensis seedlings with various concentrations of $A B T(P<0.05)$. The difference in biomass among $T$. chinensis seedlings with various concentrations of $A B T$ was nonsignificant $(P>0.05)$ under mild salt stress $(S c \leq 0.3 \%)$ but significant under moderate and severe salt stress $(S c \geq 0.6 \%$ ). At $S c$ of $1.2 \%$, there was a significant decrease in the biomass of $T$. chinensis cuttings with each concentration of $\mathrm{ABT}(P<0.05)$.

The difference in height between $T$. chinensis seedlings became significant as $S c$ increased $(P<0.05)$. Overall, the height of $T$. chinensis seedlings decreased as Sc increased (Figure 2B). Under mild (0.3\%) and moderate $(0.6 \%)$ salt stress, there was no significant difference in height between $T$. chinensis seedlings with $\mathrm{ABT}$ at 100 and $200 \mathrm{mg} \cdot \mathrm{L}^{-1}$. At $S \mathrm{C}$ of $0.9 \%$, the height of $T$. chinensis seedlings with ABT at 0,50 , 100 , and $200 \mathrm{mg} \cdot \mathrm{L}^{-1}$ was only $60.45 \%, 62.61 \%, 52.66 \%$, and $52.42 \%$ of that in the CK group. The root length of $T$. chinensis seedlings with $A B T$ at 50 and $100 \mathrm{mg} \cdot \mathrm{L}^{-1}$ first decreased and then increased as SC increased, reaching their respective minimum values at $S C$ of $0.6 \%$. At $S C$ of $0.3 \%$ and $0.6 \%$, the root length of $T$. chinensis seedlings with $\mathrm{ABT}$ at $0 \mathrm{mg}^{\cdot \mathrm{L}^{-1}}$ was significantly greater than that of $T$. chinensis seedlings with any other concentration of $\mathrm{ABT}$ (Figure $2 \mathrm{C}$ ).

\section{Chl content in T. chinensis cuttings}

The differences in Chla (Figure 3A), Chlb (Figure 3B), and ChIT (Figure 3C) contents between T. chinensis seedlings with $\mathrm{ABT}$ at various concentrations became significant $(P<0.05)$ as the salt stress level 
increased. At Sc of $0.3 \%$, the Chla, Chlb, and ChIT contents of the leaves of T. chinensis seedlings presoaked in water were lower than those in the CK group, although the differences were nonsignificant ( $P>0.05$ ). At $S c$ of $0.6 \%$, the Chla, Chlb, and ChlT contents all reached their respective peak values. At $S c$ of $0.9 \%$, the Chla and ChIT contents of the leaves of the T. chinensis seedlings reached their respective maximums, whereas their Chlb content began to decrease. As the salt stress level continued to increase, the $\mathrm{Chl}$ content in the leaves of $T$. chinensis seedlings began to decrease, suggesting that $T$. chinensis photosynthesis is enhanced by increases in Chl content to adapt to salt stress within a certain range.

At Sc $\leq 0.3 \%$, there was no difference in Chl content among T. chinensis seedlings. At Sc of $1.2 \%$, the Chla, $\mathrm{Chlb}$, and ChIT contents of the leaves of $T$. chinensis seedlings all first decreased and then increased. At Sc of $0.9 \%$, the Chla, Chlb, and ChlT contents of the leaves of $T$. chinensis seedlings all first increased and then decreased as the ABT concentration increased. Additionally, the ChIT content in T. chinensis seedlings with $A B T$ at 50,100 , and $200 \mathrm{mg} \cdot \mathrm{L}^{-1}$ was $26.05 \%, 23.04 \%$, and $11.75 \%$ higher than that of $T$. chinensis seedlings pre-soaked in water, respectively. This result suggests that under severe salt stress, ABT at suitable concentrations ( 50 and $100 \mathrm{mg} \cdot \mathrm{L}^{-1}$ ) improves $\mathrm{Chl}$ synthesis in $T$. chinensis and reduces the inhibitory effects of salt stress on Chl synthesis.

\section{SOD activity in T. chinensis cuttings}

As demonstrated in Figure 4, SOD activity in the leaves of $T$. chinensis seedlings with ABT at various concentrations first increased and then decreased with increasing salt stress. Additionally, under various salt stress conditions, SOD activity was higher in the leaves of ABT-pre-treated $T$. chinensis seedlings than in the CK group. At $S C \leq 0.6 \%$, SOD activity significantly decreased in the $T$. chinensis seedlings with an increasing $A B T$ concentration. At $S c \geq 0.9 \%$, SOD activity first increased and then decreased as the ABT concentration increased. This finding suggested that under mild salt stress, ABT increased SOD activity. As the salt stress level increased, ABT at a suitable concentration $\left(100 \mathrm{mg} \cdot \mathrm{L}^{-1}\right)$ increased SOD activity in T. chinensis seedlings. However, under severe salt stress, a high ABT concentration led to a significant decline in SOD activity.

The Sc corresponding to the peak SOD activity varied between the $T$. chinensis seedlings with ABT at various concentrations. The maximum SOD activity in the T. chinensis seedlings with $A B T$ at $0 \mathrm{mg} \cdot \mathrm{L}^{-1}$ occurred at $S C$ of $0.6 \%$ and was 5.96 times higher than in the CK group. The maximum SOD activity in $T$. chinensis seedlings with $A B T$ at 50,100 , and $200 \mathrm{mg} \cdot \mathrm{L}^{-1}$ occurred at $S C$ of $0.9 \%$ and was elevated 2.60 , 4.19 , and 4.29 times compared with the CK group, respectively. At $S c$ of $0.9 \%$, SOD activity was significantly different in $T$. chinensis seedlings with $\mathrm{ABT}$ at 50 and $100 \mathrm{mg} \cdot \mathrm{L}^{-1}(P<0.05)$ compared with the $T$. chinensis seedlings pre-soaked in water. Specifically, SOD activity was $20.85 \%$ lower and $14.72 \%$ higher in the T. chinensis seedlings with $\mathrm{ABT}$ at 50 and $100 \mathrm{mg} \cdot \mathrm{L}^{-1}$ than in the $T$. chinensis seedlings presoaked in water, respectively.

\section{POD activity in T. chinensis cuttings}


In $T$. chinensis seedlings with ABT at 50 and $200 \mathrm{mg} \cdot \mathrm{L}^{-1}, \mathrm{POD}$ activity first increased and then decreased with increasing salt stress and reached a respective maximum value at $S c$ of $0.9 \%$ (Figure 5). POD activity was relatively low in $T$. chinensis seedlings pre-treated with $A B T$ at 50 and $200 \mathrm{mg}^{-1} \mathrm{~L}^{-1}$ in the CK group. As the salt stress level increased, there was a consistent increase in POD activity in $T$. chinensis seedlings with $A B T$ at $100 \mathrm{mg} \cdot \mathrm{L}^{-1}$. However, there was no significant difference in POD activity under severe salt stress $(P>0.05)$. At $S c \leq 0.9 \%, P O D$ activity remained relatively high in the leaves of the $T$. chinensis seedlings pre-soaked in water. However, at $S c \leq 0.6 \%$, there was no significant difference in POD activity between $T$. chinensis seedlings ( $P>0.05$ ). At $S C$ of $0.3 \%, 0.6 \%, 0.9 \%$, and $1.2 \%$, POD activity was $37.20 \%, 77.52 \%, 125.35 \%$, and $56.17 \%$ higher, respectively, in the leaves of $T$. chinensis seedlings with $A B T$ at $200 \mathrm{mg} \cdot \mathrm{L}^{-1}$ than in the $\mathrm{CK}$ group. This finding suggests that salt stress significantly increased POD activity in the leaves of $T$. chinensis seedlings with $A B T$, whereas there was a relatively nonsignificant difference in POD activity between T. chinensis seedlings pre-soaked in water.

POD activity first decreased and then increased with an increasing ABT concentration at each salt stress level, except for severe salt stress $(S C=1.2 \%)$, at which there was a gradual increase in POD activity. There was a significant decrease in POD activity in $T$. chinensis seedlings with $A B T$ at suitable concentrations (50 and $100 \mathrm{mg} \cdot \mathrm{L}^{-1}$ ). By contrast, there was a significant increase in POD activity in $T$. chinensis seedlings with water or a high concentration of ABT. These findings suggest that a suitable concentration of $A B T$ can significantly reduce POD activity, whereas an overly low or high concentration of $A B T$ can easily lead to an increase in POD activity. At $S c$ of $0.6 \%-0.9 \%$, there was no significant difference in POD activity between T. chinensis seedlings with water and ABT at $200 \mathrm{mg} \cdot \mathrm{L}^{-1}(P>0.05)$, whereas there was a significant difference in POD activity between $T$. chinensis seedlings with various concentrations of ABT $(P<0.05)$. At SC of $0.6 \%, P O D$ activity was $60.86 \%$ and $40.03 \%$ higher in $T$. chinensis seedlings with $\mathrm{ABT}$ at $200 \mathrm{mg} \cdot \mathrm{L}^{-1}$ than in those pre-treated with $\mathrm{ABT}$ at 50 and $100 \mathrm{mg} \cdot \mathrm{L}^{-1}$, respectively. At SC of $0.9 \%$, POD activity was $63.12 \%$ and $53.12 \%$ higher in $T$. chinensis seedlings with $\mathrm{ABT}$ at $200 \mathrm{mg} \cdot \mathrm{L}^{-1}$ than in those pre-treated with $\mathrm{ABT}$ at 50 and $100 \mathrm{mg} \cdot \mathrm{L}^{-1}$, respectively.

\section{MDA content in $T$. chinensis cuttings}

As the salt stress level increased, the MDA content first increased and then decreased in $T$. chinensis cuttings with $\mathrm{ABT}$ at 0,50 , and $200 \mathrm{mg} \cdot \mathrm{L}^{-1}$. In comparison, as the salt stress level increased, there was a gradual increase in the MDA content in T. chinensis cuttings with ABT at $100 \mathrm{mg} \cdot \mathrm{L}^{-1}$ (Figure 6). This result may be a consequence of enzyme system regulation. Owing to this regulation, there was an increase in activities of relevant antioxidant enzymes. These enzymes, in turn, reduced the cell membranes damage caused by salt stress. The MDA content in leaves of $T$. chinensis cuttings presoaked in water reached a maximum $\left(30.21 \mathrm{nmol} \cdot \mathrm{mg}^{-1} \mathrm{prot}\right)$ at $S \mathrm{C}$ of $0.6 \%$. At this $\mathrm{Sc}$, the leaves of the $T$. chinensis cuttings sustained significant damage caused by membrane lipid peroxidation (MLP).

At the same $S c$, the MDA content in ABT-pre-treated T. chinensis seedlings exhibited various patterns at different ABT concentrations. At Scs of $0.6 \%$ and $0.9 \%$, the MDA content first decreased and then 
increased. Additionally, at these two Scs, the MDA content was significantly higher in T. chinensis seedlings pre-soaked in water than in $T$. chinensis seedlings pre-treated with ABT. At $S c$ of $1.2 \%$, the MDA content first increased and then decreased, suggesting that at a suitable concentration (50 and 200 $\left.\mathrm{mg} \cdot \mathrm{L}^{-1}\right), \mathrm{ABT}$ prevented T. chinensis seedlings from being damaged by severe salt stress and led to a significant decrease in their MDA content.

\section{Discussion}

\section{Effects of salt stress on growth conditions of T. chinensis}

The survival rate of $T$. chinensis cuttings under mild salt stress (Sc $\leq 0.3 \%)$ was relatively high and differed nonsignificantly from that of $T$. chinensis cuttings in the CK group. This difference suggests that $T$. chinensis is tolerant to salt to a certain extent. As the salt stress level increased, the survival rate of $T$. chinensis cuttings became relatively low or even zero ( $S c>1.2 \%$ ). Increasing salt stress also caused increasing damage in $T$. chinensis cuttings and significantly affected the normal growth of their root systems. The effects of growth regulator concentrations on cutting growth vary relatively significantly. An increase in the growth regulator concentration will prevent gymnosperms from rooting. Some research has also found that increasing the growth regulator concentration will not increase the rooting percentage but can improve the quality of the root system. This phenomenon is related to the difference in the type and concentration of hormones needed and the hormone sensitivities among tree species[35]. A rooting powder (e.g., ABT) can be used to facilitate growth of the root system of T. chinensis cuttings. By increasing and regulating the content of endogenous hormones and the activities of important enzymes in $T$. chinensis seedlings, ABT stimulates cell division and growth in the inner root sheath, strengthens root system development, facilitates vigorous growth, significantly reduces cell membrane damage caused by salt stress, and increases salt stress adaptability. In this study, under mild salt stress (Sc $\leq 0.3 \%$ ), the ABT concentration showed no significant impact on the survival rate of $T$. chinensis cuttings. As $S c$ increased, ABT at a suitable concentration increased the survival rate. However, as the salt stress level continued to increase, $A B T$ became ineffective. A relatively high soil salinity generally causes osmotic stress in plants and disrupts their nutrient ion balance, thereby affecting physiological and biochemical processes such as growth, photosynthesis, osmotic adjustment substance synthesis, and lipid metabolism[36], ultimately limiting their growth rate and biomass accumulation. In this study, there was a significant decrease in the biomass, root length, and height of T. chinensis under salt stress. T. chinensis may reduce biomass accumulation and utilize more resources and energy to respond to high salinity-induced damage[36], which suggests that plants under stress are able to respond to adverse external conditions by altering their biomass allocation pattern. By reducing the proportion of biomass allocated to root systems, some plants reduce salt absorption and salt transport to their aboveground portions[37]. Some plants acquire more water and nutrients by increasing the proportion of biomass allocated to their root systems. This mechanism increases plant growth ability and salt dilution in plant cells[38]. In this study, T. chinensis seedlings pre-treated with ABT at $100 \mathrm{mg} \cdot \mathrm{L}^{-1}$ had relatively long root 
systems and the highest biomass at Sc of $0.9 \%$, and their aboveground and belowground portions showed consistent growth.

\section{Effects of salt stress on $\mathrm{Chl}$ contents in T. chinensis cuttings}

$\mathrm{Chl}$ is an important substance in plant photosynthesis. The Chl content can reflect the ability of plants to assimilate substances. Under salt stress, plant Chl contents gradually decreased as Sc increased. Some research has shown that as $S c$ increases, the $\mathrm{Chl}$ content in plants either first increases and then decreases or gradually increases, and the plants exhibit relatively high salt tolerance[39]. In this study, as Sc increased, Chla, Chlb, and ChIT contents in the leaves of T. chinensis cuttings pre-treated with ABT at various concentrations all initially decreased, then increased, and then decreased again. This pattern may result from the low Scs, which was conducive to $T$. chinensis growth and did not activate its resistance mechanism to salt stress. As the salt stress level increased, the Chl content began to increase. Because Chl synthesis requires Pro, under low salt stress, a large amount of Pro accumulated in the leaf cells of $T$. chinensis, facilitating $\mathrm{Chl}$ synthesis. T. chinensis photosynthesis in leaves is enhanced by increases in Chl synthesis to adapt to salt stress. Under high salt stress, a decrease in the Chl content was observed herein, suggesting an increase in the inhibitory effects of salt stress on $T$. chinensis. This phenomenon resulted in a decrease in the content of glutamic acid, the precursor for the synthesis of 5-aminolevulinic acid (5-ALA), in leaves and consequently in 5-ALA content. These changes ultimately limited Chl synthesis, for which 5-ALA is the precursor and, to some extent, damaged the Chl synthesis system. Additionally, salt stress facilitated the decomposition of $\mathrm{Chl}$ by chlorophyllase[40], resulting in a decrease in Chl content. These alterations were similar to the changes in Chl content in T. chinensis seedlings[16] and in Rhaphiolepis umbellata, Rosa chinensis, and Morus alba[41] under salt stress. The Scs corresponding to the peak $\mathrm{Chl}$ content differed in leaves of $T$. chinensis pre-treated with $\mathrm{ABT}$ at various concentrations. The higher the ABT concentration was, the higher the $S c$ corresponding to the peak $\mathrm{Chl}$ content in T. chinensis leaves, which suggested that applying ABT at a specific concentration could reduce the effects of salt stress on Chl synthesis in $T$. chinensis cuttings, potentially because ABT can increase the $T$. chinensis rooting rate and activity and thereby allows its relatively early adaptation to salt stress.

\section{Effects of salt stress on the activities of antioxidant enzymes in $T$. chinensis cuttings}

Salt stress leads to an increase in reactive oxide species (ROS) levels in plants. ROS affect many cell functions by damaging nucleic acids, oxidizing proteins, and causing lipid peroxidation[42]. Plants have an ROS removal system consisting of antioxidant enzymes as key components. SOD and POD are the primary antioxidant enzymes in plants under salt stress and play a vital role in removing superoxide ions, preventing MLP, and reducing plasma membrane damage[11]. Under normal conditions, there is a dynamic balance between the production and removal of ROS in cells. Under stress, this balance is disrupted, and ROS start to accumulate. Plants remove excess ROS by increasing SOD activity. POD and 
SOD collectively remove ROS. SOD converts the ROS formed in the oxidation process into oxygen and $\mathrm{H}_{2} \mathrm{O}_{2}$ through disproportionation reactions. Subsequently, POD decomposes and removes the formed $\mathrm{H}_{2} \mathrm{O}_{2}$ [11]. Under salt stress, there is a threshold for the ROS level tolerable to plant cells. Below this threshold, plants are able to remove ROS by increasing the activities of antioxidant enzymes. Beyond this threshold, the activities of antioxidant enzymes will be inhibited, resulting in an excess accumulation of ROS and leading to plant tissue damage[28]. In this study, as the salt stress level increased, SOD activity first increased and then decreased in the leaves of the $T$. chinensis cuttings pre-treated with ABT at various concentrations. This result suggested that as the salt stress level increased, ROS began to accumulate in the leaf cells of $T$. chinensis, and $T$. chinensis removed excess ROS by increasing the SOD activity to adapt to salt stress. However, under relatively high salt stress, the ROS that formed exceeded the regulation ability of SOD. Consequently, SOD activity was inhibited and therefore decreased. This SOD pattern is consistent with that in T. austromongolica and T. chinensis[21] in saline and alkaline habitats and in the leaves of Pennisetum alopecuroides (L.) Spreng[43] under $\mathrm{NaCl}$ stress.

Plants reduce salt stress-induced damage by increasing POD activity. The causes of the increase in POD activity include not only ROS production but also cell membrane damage and changes in the calcium ion concentration. At various SCS, POD activity in the leaves of $T$. chinensis cuttings pre-soaked in water was relatively high, suggesting that the relatively large amount of $\mathrm{H}_{2} \mathrm{O}_{2}$ produced in leaf cells of $T$. chinensis seedlings that were not pre-treated with ABT resulted in increased POD activity to remove $\mathrm{H}_{2} \mathrm{O}_{2}$. Evidently, the damage (MLP) induced by salt stress was three times greater in T. chinensis seedlings that were not pre-treated with ABT than in T. chinensis seedlings in the three ABT pretreatment groups. Li et al. found that as the salt stress level was increased, POD activity in T. austromongolica and T. chinensis first increased and then decreased[21]. They also found that POD activity in the leaves of $T$. austromongolica and $T$. chinensis reached a maximum at $S C$ of $1.2 \%$, which was 3.5 and 3.6 times greater than in the control group, respectively. A similar pattern was also found in this study. POD activity in the leaves of $T$. chinensis seedlings pre-treated with $A B T$ at $200 \mathrm{mg} \cdot \mathrm{L}^{-1}$ first increased and then decreased with increasing salt stress. At SCs of $0.3 \%-0.9 \%$, there was a significant increase in POD activity in the leaf cells. The antioxidant enzyme system decomposed the $\mathrm{H}_{2} \mathrm{O}_{2}$ produced by SOD through disproportionation reactions by increasing POD activity. Under high salt stress ( $S C \geq 1.2 \%$ ), excess ROS were produced in leaf cells, exceeding the removal threshold of POD. As a result, the accumulating ROS damaged the enzyme system, resulting in a decline in POD activity. Compared with pretreatment with $\mathrm{ABT}$ at 50 and $100 \mathrm{mg} \cdot \mathrm{L}^{-1}$, the sensitivity of POD activity was relatively low in the leaves of $T$. chinensis seedlings pre-treated with $A B T$ at $200 \mathrm{mg} \cdot \mathrm{L}^{-1}$, and the ability of POD in these T. chinensis seedlings to regulate salt tolerance was also relatively low.

\section{Effects of salt stress on MDA content in T. chinensis cuttings}

Plants produce MDA as a result of MLP under stress. MDA is the main indicator of cell membrane damage and free radical formation in plants. As MDA increasingly accumulates, membrane damage 
increases and the resistance of the plant declines. Thus, MDA can be used as an important index for evaluating the extent of membrane system damage under stress[44]. The MDA content in plant leaves varies relatively significantly with their salt tolerance. As salt stress increases, there is a continuous increase in MDA content in the leaves of plants with relatively low salt tolerance, such as $T$. chinensis tissue culture seedlings[45]. In comparison, with increasing salt stress, the MDA content in the leaves of plants with relatively high salt tolerance first decreases and then increases[32]. In this study, the MDA content in the leaves of $T$. chinensis cuttings first increased and then decreased as the salt stress level increased. This finding is consistent with those reported by Li et al. showing the effects of MLP in the seedling leaves of six gramineous forages[46], but differs from the results of Qiu et al. investigating the MDA content in Lagerstroemia indica 'Pink Velour' under salt stress[47]. This distinction may be related to the relatively significant differences in salt stress tolerance regulatory mechanisms among plants. Here, MDA content in the leaves of $T$. chinensis peaked at Sc of $0.6 \%$ and then decreased at SC of $0.9 \%$; SOD and POD activities were relatively high at $S c$ of $0.9 \%$. These findings suggest that through inter-regulation, SOD and POD can effectively remove the excess ROS produced under salt stress and maintain the number of ROS in the organism at a low level, thereby essentially preventing ROS-induced MLP and other damaging processes. However, under severe salt stress $(S C=1.2 \%)$, SOD and POD activities were both relatively low, and there was a significant decrease in MDA content, which might be related to the adaptive regulation of a certain dominant factor under the combined action of salt stress and $A B T$. The relevant internal mechanism requires further analysis.

\section{Conclusions}

As the salt stress level increased, the survival rate, root length, and height of $T$. chinensis cuttings gradually decreased, whereas their biomass first increased and then decreased. At $S_{c} \geq 1.2 \%$, there was a significant decrease in $T$. chinensis cutting biomass, and the survival rate was zero. $A B T$, the rooting powder used in this study, enhanced the resistance of $T$. chinensis to severe salt stress $(S c>0.6 \%)$. ABT treatment exhibited a relatively high compensatory effect on $T$. chinensis cuttings under salt stress. ABT at a concentration of $100 \mathrm{mg} \cdot \mathrm{L}^{-1}$ exhibited a positive effect on the growth of $T$. chinensis. The effectiveness of $A B T$ in improving the survival rate and growth of $T$. chinensis cuttings became increasingly pronounced as the salt stress level increased. ABT was most effective in improving the survival rate of $T$. chinensis when applied at 50 and $100 \mathrm{mg} \cdot \mathrm{L}^{-1}$.

T. chinensis seedling photosynthesis was enhanced by increasing the content of $\mathrm{Chl}$ to adapt to salt stress at suitable levels. However, under excessively high salt stress, Chl synthesis was disrupted, resulting in a decline in $\mathrm{Chl}$ content. Increasing the ABT concentration could strengthen the tolerance of $T$. chinensis seedlings to salt stress during $\mathrm{Chl}$ synthesis.

As the salt stress level increased, T. chinensis seedlings showed reduced ROS-induced damaged through increases in SOD and POD activities. Excess ROS accumulation inhibited the increase in enzyme activities. More significant changes in MLP in the cell membranes of $T$. chinensis seedlings resulted in higher MDA accumulation. ABT helped enhance the regulatory ability of the enzyme system of $T$. 
chinensis cuttings and significantly reduced the damage caused by low salt stress in cell membranes. The enzyme activities were highest in the $T$. chinensis seedlings pre-treated with $A B T$ at $100 \mathrm{mg} \cdot \mathrm{L}^{-1}$, and the cell membranes in these seedlings sustained the least significant oxidative damage. The following conditions were found to be suitable for vegetative propagation of T. chinensis: $S c \leq 0.9 \%$ and $A B T \leq 100$ $\mathrm{mg} \cdot \mathrm{L}^{-1}$. At SC of $0.9 \%$, T. chinensis seedlings pre-treated with $\mathrm{ABT}$ at $100 \mathrm{mg} \cdot \mathrm{L}^{-1}$ showed the most vigorous growth, had the highest biomass, and exhibited relatively high physiological regulatory ability and salt adaptability.

\section{Abbreviations}

$A B T$, aminobenzotriazole; $S C$, soil salinity; Chl, chlorophyll; SOD, superoxide dismutase; POD, peroxidase; MDA, malondialdehyde; ROS, reactive oxide species; MLP, membrane lipid peroxidation; CAT, catalase; Chla, chlorophyll-a; Chlb, chlorophyll-b; ChIT, total Chl; 5-ALA, 5-aminolevulinic acid.

\section{Declarations}

\section{Acknowledgements}

The authors thank Pro. Jingkuan Sun, Dr. Jinfang Zhu and Ms. Ying Fang for their assistance during the experiments.

\section{Funding}

The research was financially supported by the National Key Research and Development Program of China (No.2017YFC0505904); the National Natural Science Foundation of China (No.31770761) and Taishan Scholars Program of Shandong Province, China.

\section{Availability of data and materials}

The datasets during or analysed during the current study available from the corresponding author on reasonable request.

\section{Authors'contribution}

Jia Sun, the first author of the paper, had overall responsibility for the experimental design, data collection, analysis, writing and project management; Jiangbao Xia is the corresponding author, and made significant contributions to experimental arrangements and manuscript preparation; Ximei Zhao and Li Su made significant contributions to data collection and analysis; Chuanrong Li and Fanglei Gao made significant contributions to the interpretation of the data and manuscript preparation; Ping Liu made significant contributions to data collection and analysis and writing. 


\section{Competing interests}

The authors declare that they have no conflict of interest.

\section{Consent for publication}

Not applicable.

\section{Ethics approval and consent to participate}

Not applicable.

\section{Deposition of data}

Not applicable

\section{Author details}

${ }^{1}$ College of Forestry, Shandong Agricultural University, Taian, Shandong 271018

${ }^{2}$ Binzhou University, Shandong Key Laboratory of Eco-Environmental Science for the Yellow River Delta, Binzhou 256603, China

\section{References}

1. Zhou D, Lin ZL, Liu LM. Regional land salinization assessment and simulation through cellular automaton-Markov modeling and spatial pattern analysis. Science of the Total Environment. 2012;439(11): 260-274.

2. Lin J, Dilbar S. Progress in the study on soil salinization. Journal of Xinjiang University (Natural Science Edition). 2007;24(3):318-323.

3. Wang JL, Huang XJ, ZhongTY, Chen ZG. Review on sustainable utilization of salt-affected land. Acta Geographica Sinica. 2011;66(5):673-684.

4. Glenn EP, Nelson SG, Ambrose B, Martinez K, Soliz D. Comparison of salinity tolerance of three Atriplex SPP. in well-watered and drying soil. Environmental and Experimental Botany. 2012;83:6272.

5. Qadir M, Ghafoor A, Murtaza G. Amelioration strategies for saline soils: A review. Land Degradation \& Development. 2000;11(6):501-521. 
6. Hasanuzzaman M, Nahar K, Alam MM, Bhowmik PC, Hossain MA, Rahman MM, Prasad MN, Ozturk M, Fujita M. Potential use of halophytes to remediate saline soils. Journal of Biomedicine and Biotechnology. 2014;(8):589341.

7. Bhuiyan MSI, Raman A, Hodgkins D, Mitchell D, Nicol HI. Influence of high levels of $\mathrm{Na}^{+}$and $\mathrm{Cl}^{-}$on ion concentration, growth, and photosynthetic performance of three salt-tolerant plants. Flora. 2017;228:1-9.

8. Yang C, Chen HY, Li JS, Tian Y, Feng XH, Liu XJ, Guo K. Soil improving effect of Suaeda salsa on heavy coastal saline-alkaline land. Chinese Journal of Eco-Agriculture. 2019;27(4):105-113.

9. Kalaji HM, Govindjee, Bosa K, Kościelniak J, Żuk-Gołaszewska K. Effects of salt stress on photosystem II efficiency and $\mathrm{CO}_{2}$ assimilation of two syrian barley landraces. Environmental and Experimental Botany. 2011;73:64-72.

10. Luo D, Shi YJ, Song FH, Li JC. Effects of salt stress on growth, photosynthetic and fluorescence characteristics,and root architecture of Corylus heterophylla $\times$ C. avellan seedlings. Chinese Journal of Applied Ecology. 2019;43(5):178-185.

11. Farhangiabriz S, Torabian S. Antioxidant enzyme and osmotic adjustment changes in bean seedlings as affected by biochar under salt stress. Ecotoxicology \& Environmental Safety. 2017; 137:64-70.

12. Xue YF, Liu ZP. Antioxidant enzymes and physiological characteristics in two Jerusalem artichoke cultivars under salt stress. Russian Journal of Plant Physiology. 2008;55(6):776-781.

13. Hu G, Liu Y, Duo T. Antioxidant metabolism variation associated with alkali-salt tolerance in thirty switchgrass (Panicum virgatum) lines. Plos One. 2018;13(6):407.

14. Li BB, Ouyang J, Wang JY, Wu HF, Liu XJ, Zou JH. Effects of NaCl on seedling growth and some physiological characteristics of Salix matsudana Koidz. Journal of Tianjin Normal University (Natural Science Edition). 2017;37(6):37-42.

15. Hong WJ, Shen CQ, Zhuang XY. Effect of NaCl Stress on Physiological Responses and Anatomical Structure of Salix spp. Seedlings. Journal of Tropical and Subtropical Botany. 2017;25(5):489-496.

16. Zhu JF, Liu JT, Lu ZH, Xia JB, Liu HN, JinY. Effects of salt stress on physiological characteristics of Tamarix chinensis Lour. Seedlings. Acta Ecologica Sinica. 2015;35(15):5140-5146.

17. Zhu JF, Xia JB, Lu ZH, Liu JT, Sun JK. Growth, Physiological and Biochemical Characteristics of Tamarix chinensis Seedlings under Salt-drought Intercross Stress. Acta Bot. Boreal.- Occident. Sin. 2012;32(1):124-130.

18. Feng Y, Yin K. Study on organs morphology and its taxonomic significance of Tamarix L.. Arid Zone Research. 2000;17(3):40-45.

19. Zhao FX, Yin LK. Spatial distribution pattern and interspecific association of Popolus euphratica and Tamarix ranosissima seedlings populations along desert inland river. Chinese Journal of Ecology. 2007;26(7):972-977. 
20. Yang CP, Wang YC, Liu GF, Jiang J. Study on gene expression of Tamarix under $\mathrm{NaHCO}_{3}$ stress using SSH technology. Acta Genetica Sinica. 2004;31(9):926-933.

21. Li YT, Wang X, Wei HX, Yang QS, Zhou J, Liu DX, Liu ZJ, Wei WQ. Physiological Characteristics of

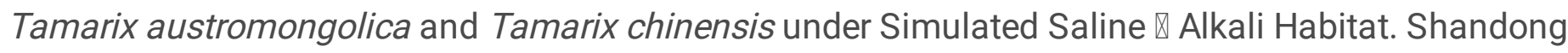
Agricultural Sciences. 2017;49(1):53-58.

22. Gaskin JF, Schaal BA. Hybrid Tamarix widespread in U.S. invasion and undetected in native Asian range. Proceedings of the National Academy of Sciences. 2002;99(17):11256-11259.

23. Gaskin JF, Kazmer DJ. Introgression between invasive saltcedars (Tamarix chinensis and T. ramosissima) in the USA. Biological Invasions. 2009;11(5):1121-1130.

24. González E, Sher AA, Anderson RM, et al. Secondary invasions of noxious weeds associated with control of invasive Tamarix are frequent, idiosyncratic and persistent. Biological Conservation. 2017;213:106-114

25. Newete SW, Allem SM, Venter N, Byrne MJ. Tamarix efficiency in salt excretion and physiological tolerance to salt-induced stress in South Africa. Intertional Journal of Phytoremediation 1-7. https://doi.org./10.1080/15226514.2019.1633997

26. Parađiković N, Zeljković S, Tkalec M, Vinković T, Dervić I, Marić M. Influence of rooting powder on propagation of sage (Salvia officinalis L.) and rosemary (Rosmarinus officinalis L.) with green cuttings. Poljoprivreda. 2013;19(2):10-15.

27. Song SJ, Ko CH, Shin US, Oh HJ, Kim SY, Le SY. Successful stem cutting propagation of Patrinia rupestris for horticulture. Rhizosphere. 2019;9:90-92.

28. Jamal A, Ayub G, Rahman A, Rashid A, Ali J, Shahab M. Effect of IBA (Indole Butyric Acid) levels on the growth and rooting of different cutting types of Clerodendrum splendens. Pure and Applied Biology. 2016;5:64.

29. Tan GF. The experiment of Tamarix chinensis Lour冈tender branch cuttage. Journal of Jilin Forestry Science and Technology. 2014;43(6):11-13.

30. Zhang XJ, Wang Q, Yin $\mathrm{CH}$, Sheng JH. Effect of Rooting powder and Fertilizer on Root Activity of Tamarix ramosissima. Forestry Science \& Technology. 2009;34(5):14-16.

31. Li HS. The Experiment Principle and Technology of Plant Physiology. Beijing: Higher Education Press. 2000.

32. Luo GH, Wang AG. The Experiment Guide of Modern Plant Physiology. Beijing: Science Press. 1999.

33. Mao AJ, Wang YJ, Feng LX, Xu Y, Geng SS, Cao WH. Variation of polyphenoloxidase, peroxidase and phenylalanine ammonia-lyase in hot pepper seedlings infected by Phytophthora capsici L. Acta Agricultural Boreali-Sinica. 2003;18(2):66-69.

34. Zhao SJ, Li DQ. The Experiment Guide of Modern Plant Physiology. Beijing: Science Press.1999.

35. Jin JQ, Guo QS, Zhu L, Xu GX. Study on Cutting Propagation of Thuja sutchuenensis \An Endangered Species Endemic to China. Forest Research. 2013;26(1):94-100》 
36. Zhang XX, Yin XL, Li HL, Su D, Jia SY, Dong Z. Effect of salt stress on the biomass and photosynthetic characteristics of Ulmus pumila L. strains. Acta Ecologica Sinica. 2017;37(21):72587265.

37. Osone Y, Tateno M. Applicability and limitation of optimal biomass allocation models: A test of two species from fertile and infertile habitats. Annals of Botany. 2005;95:1211-1220.

38. Guo LL, Hao LH, Jia HH. Li F, Zhang QQ, Cao X, Xu M, Zheng YP. Effects of NaCl stress on stomatal traits囚leaf gas exchange parameters $₫ a n d$ biomass of two tomato cultivars. Chinese Journal of Applied Ecology. 2018;29(12):3949-3958.

39. Yang S, Zhang HX, Liu T. Morphological changes and physiological characteristics of seedlings from 16 tree species with salt stress. Journal of Zhejiang A \& F University. 2012;29(5):744-754.

40. Zhao KF. Salt-resistance physiology of plants. Beijing: China Science and Technology Press. 1993;230-231.

41. Qiu LZ, Huang YJ, Huang JQ, Xia GH, Gong N. Comparative study on vegetal and physiological characteristics of different salt-tolerant plants under salt stress. Journal of Zhejiang University (Agriculture and life science). 2006;32(4):420-427.

42. Foyer $\mathrm{CH}$, Noctor $\mathrm{G}$. Redox homeostis and antioxidant signaling: a metabolicinterface between stress perception and physiological responses. Plant Cell. 2005;17:1866-1875.

43. Miao S, Xia ZP, Li ZQ. Effect of $\mathrm{NaCl}$ on Growth and Physiological Characteristics of Three Pennisetum Species. Heilongjiang Agricultural Sciences. 2019;(6):132-136.

44. Karim S, Behrouz S, Vahid R, Mahmood K, Adriano S. Salt stress induction of some key antioxidant enzymes and metabolites in eight Iranian wild almond species $₫$ Acta Physiol Plantarum. 2012;34(1):203-213】

45. Guo NN, Chen XL, Zhang J, Chen JY, Zhu YJ, Ding YT. Changea in Antioxidase Activity and Osmotic Adjusting Substance of Tamarix chinensis Seedlings under $\mathrm{NaCl}$ Stress. Acta Bot. Boreal.- Occident. Sin. 2015;35(8):1620-1625.

46. Li Q, Liu GD, Huan SQ. The Activities of Protective Enzymes of Grass Seedlings Subjected to Long Salinity and Their Relationship to Salinity Tolerance. Aeta Ecologiae Animalis Domastiei. 2005; (5):63-67.

47. Qiu GJ, Yu M, Hu WX, Chen K, Yao ZY. Effects of salt stress on growth and physiological and biochemical characteristics of Lagerstroemia indica "Pink Velour". Jiangsu Agriculture Science. 2018;46(6):123-126.

\section{Figures}




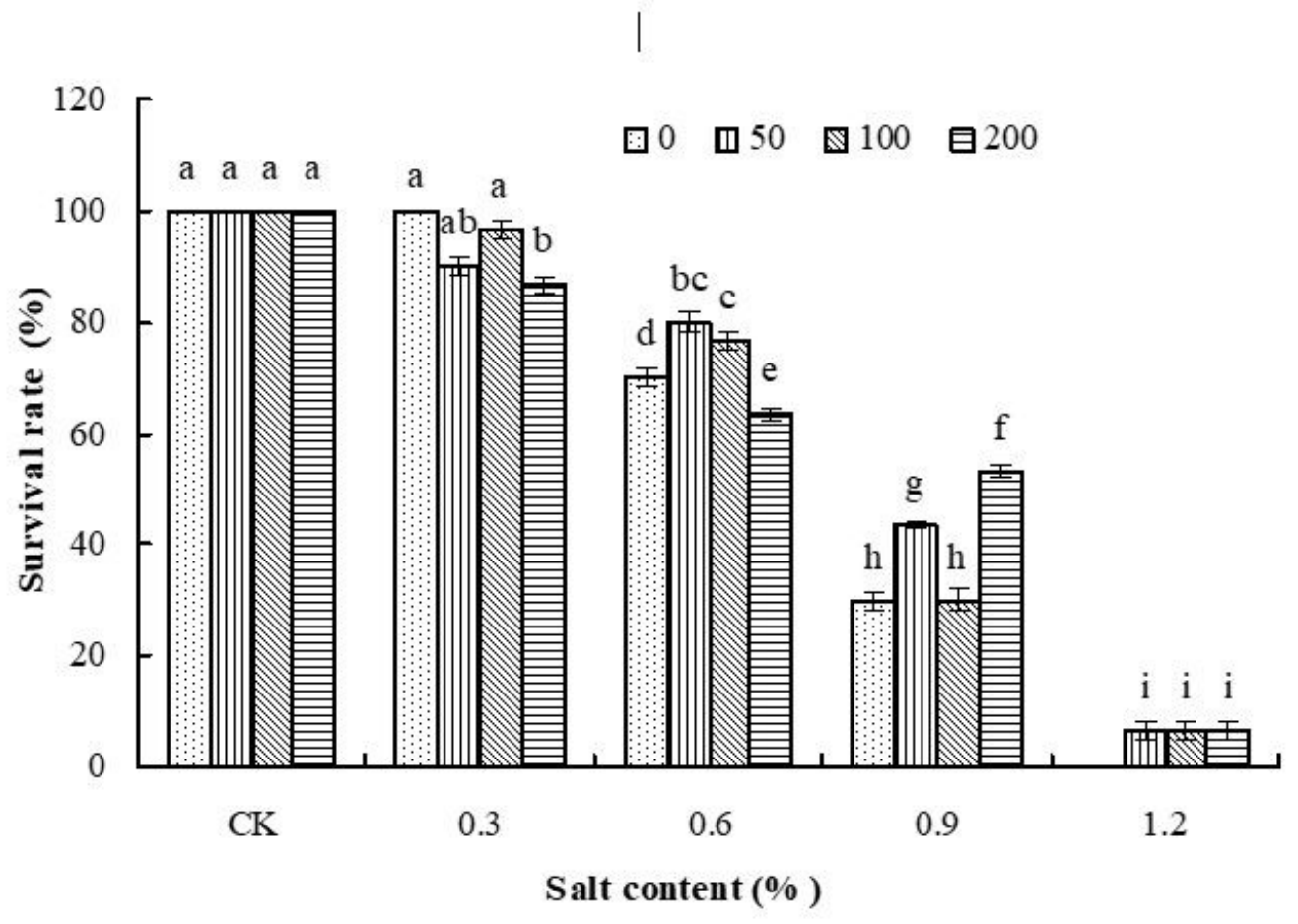

Fig.1

Figure 1

Effects of salt stress on the survival rate of $T$. chinensis cuttings with various concentrations of $A B T$ In this and all subsequent figures, different letters signify a significant difference between treatments $(P<0.05)$ 

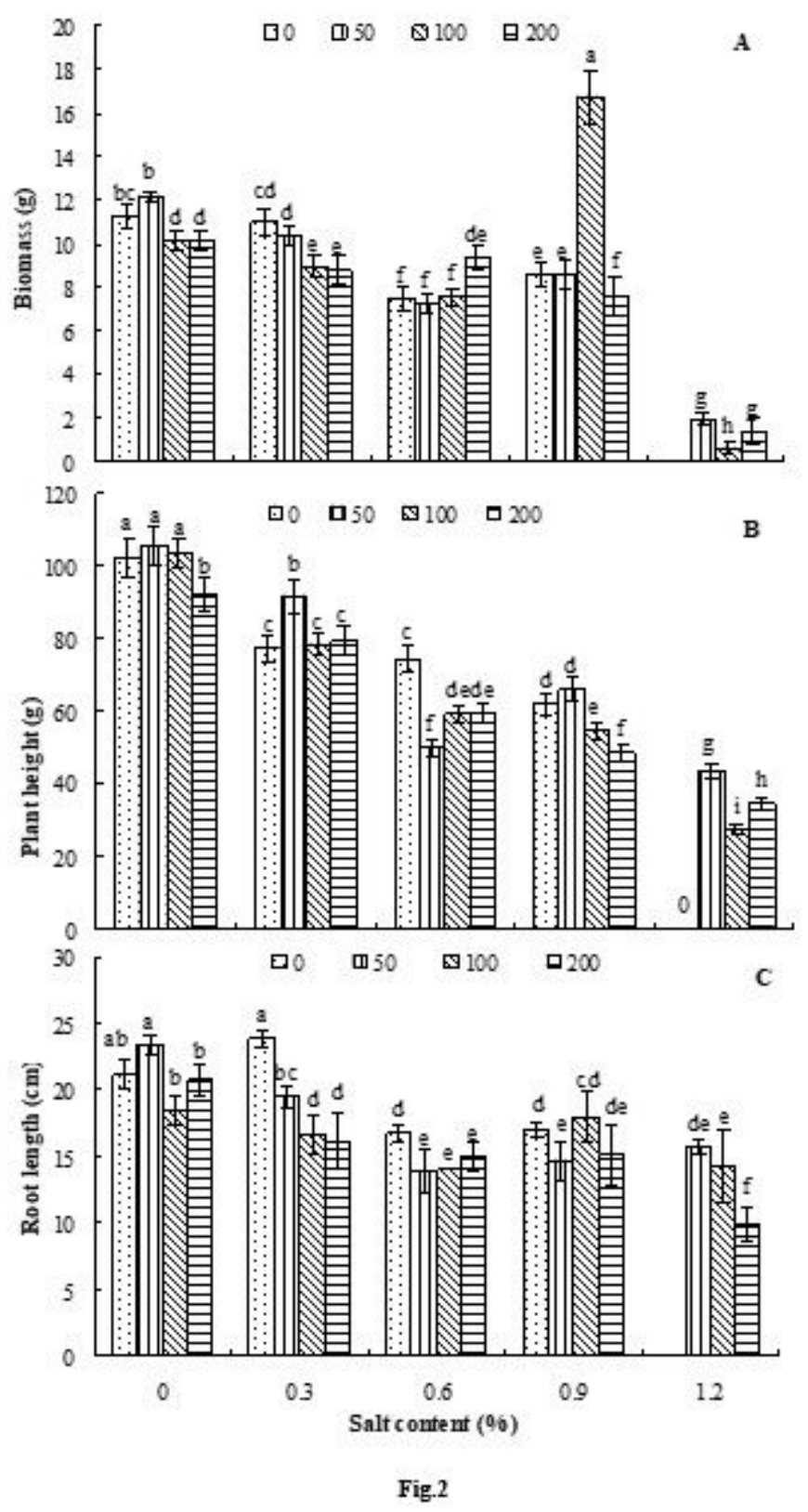

Figure 2

Effects of salt stress on the biomass of T. chinensis cuttings with various concentrations of ABT 

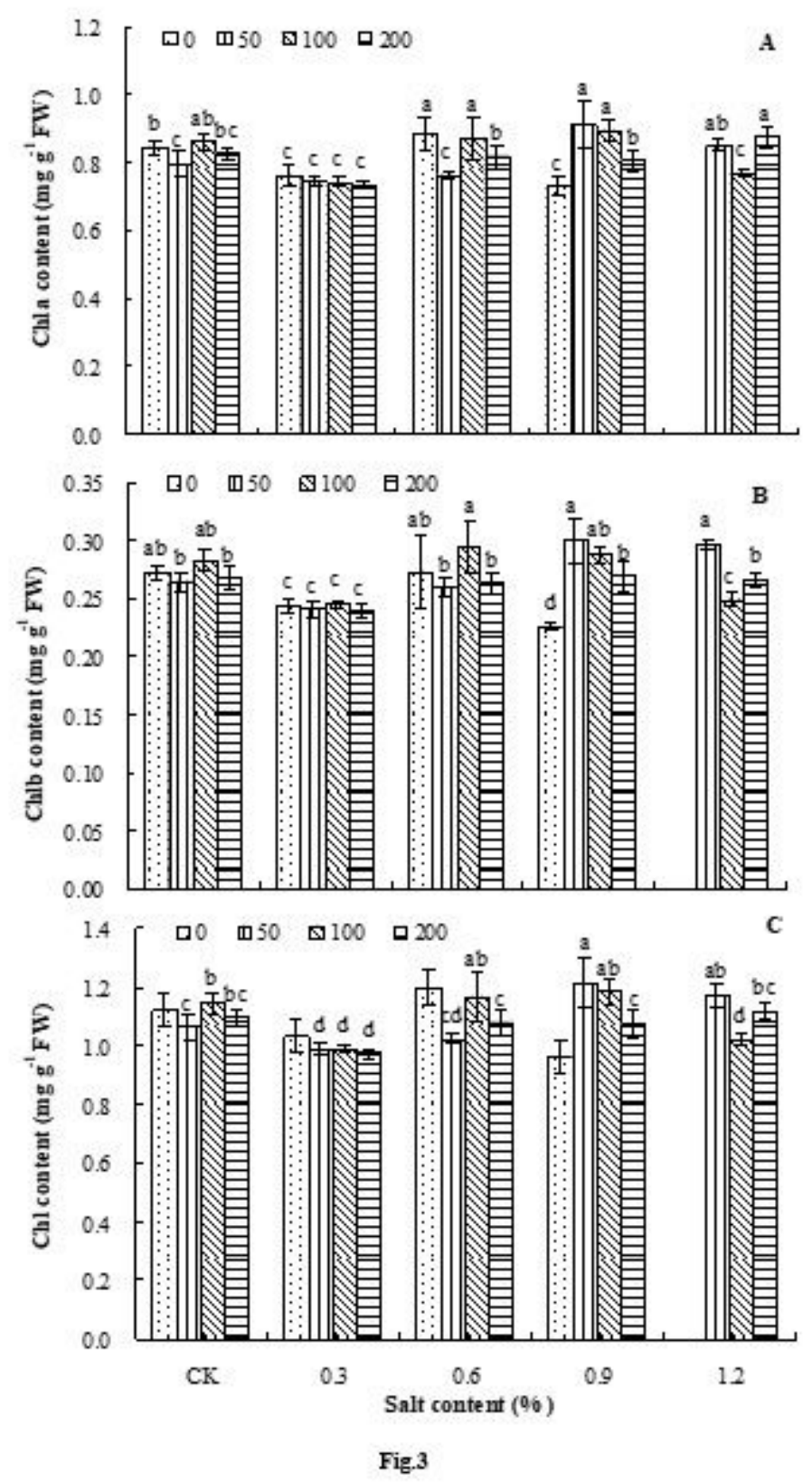

Figure 3

Effects of salt stress on the $\mathrm{Chl}$ content in the leaves of T. chinensis cuttings with various concentrations of $A B T$ 


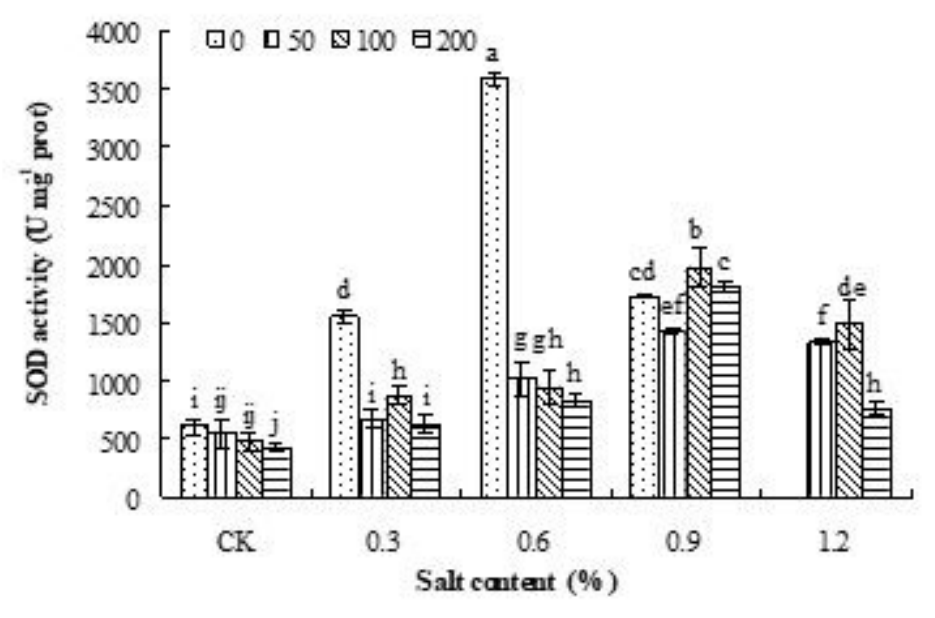

Fig.4

\section{Figure 4}

Effects of salt stress on SOD activity in the leaves of T. chinensis cuttings with various concentrations of ABT

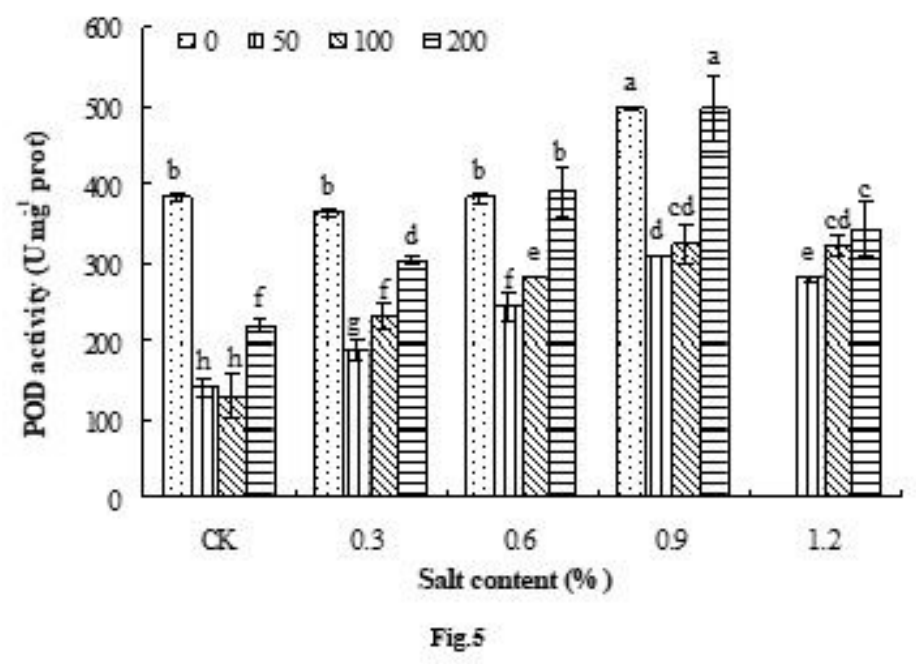

Figure 5

Effects of salt stress on POD activity in the leaves of T. chinensis cuttings with various concentrations of ABT 


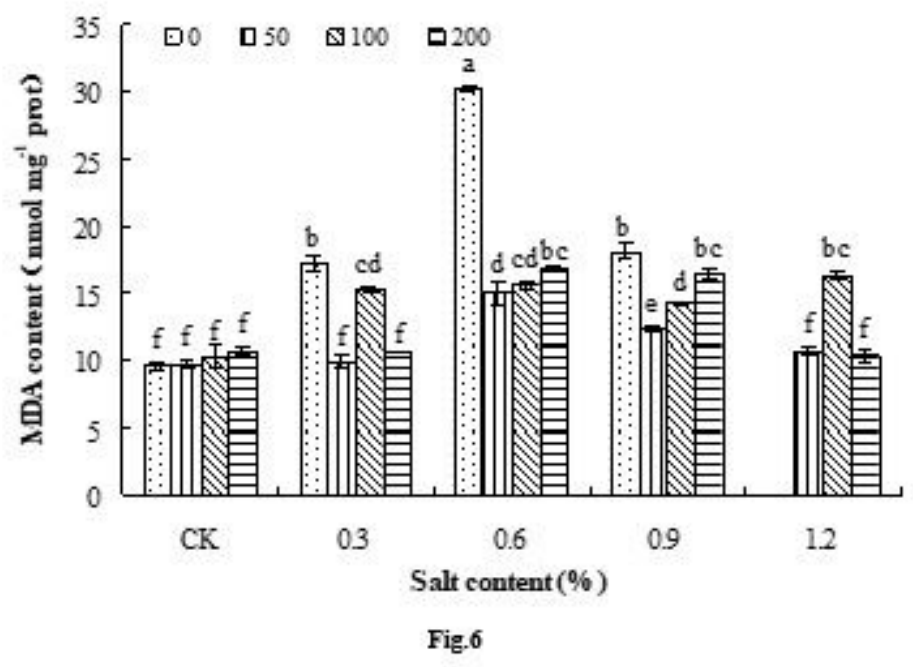

Figure 6

Effects of salt stress on MDA content in leaves of T. chinensis cuttings with various concentrations of $\mathrm{ABT}$ 